\title{
Evaluation of Molecular Species of Prostate-Specific Antigen Complexed with Immunoglobulin $M$ in Prostate Cancer and Benign Prostatic Hyperplasia
}

\author{
Sanja Goč and Miroslava Janković \\ Institute for the Application of Nuclear Energy INEP, University of Belgrade, Banatska 31b, 11080 Zemun, Serbia \\ Correspondence should be addressed to Miroslava Janković; j.miroslav@sbb.rs
}

Received 21 August 2013; Revised 4 October 2013; Accepted 5 October 2013

Academic Editor: Ferdinando Mannello

Copyright (C) 2013 S. Goč and M. Janković. This is an open access article distributed under the Creative Commons Attribution License, which permits unrestricted use, distribution, and reproduction in any medium, provided the original work is properly cited.

\begin{abstract}
This study was aimed at defining molecular species of prostate-specific antigen (PSA) in immune complexes with immunoglobulin $\mathrm{M}(\operatorname{IgM})$. Having in mind the oligoreactivity of IgM and its preference for carbohydrate antigens, there is the possibility that it can selectively recognize known PSA glycoisoforms. PSA-IgM complexes and free PSA fractions were separated from the sera of subjects with prostate cancer $(\mathrm{PCa})$ and benign prostatic hyperplasia $(\mathrm{BPH})$ by gel filtration and subjected to on-chip immunoaffinity and ion-exchange chromatography. PSA-immunoreactive species were detected using surface-enhanced laser desorption/ionization time of flight mass spectrometry. The obtained spectra were analyzed for protein and glycan composition. The general pattern of the molecular species of PCa PSA and BPH PSA found in complexes with IgM was similar. It comprised major peaks at $17 \mathrm{kDa}$ and minor peaks at $28 \mathrm{kDa}$, corresponding to the entire mature glycosylated PSA. The main difference was the presence of incompletely glycosylated $26.8 \mathrm{kDa}$ species, having putative paucimannosidic structures, observed in PCa PSA-IgM, but not in BPH PSA-IgM. Characteristic PCa PSA-IgM glycoforms pose the question of the possible role of glycosylation as a framework for immune surveillance and may be of interest in light of recent data indicating mannose-containing glycans as cancer biomarker.
\end{abstract}

\section{Introduction}

Efforts in the field of biomarker research have pointed to the existence of circulating immune complexes as a novel class of tumor markers with diagnostic potential comparable with or greater than that of the corresponding free biomarker. They include biomarker-immunoglobulin M (IgM) complexes, which have been found in several neoplastic diseases, such as colorectal, liver, and prostate cancer [1-3]. Although they are supposed to be a valuable adjunct in differential diagnostics, the biological meaning of this kind of complexes has not been elucidated; that is, it is not known whether they have any physiological role. In addition, from the biochemical point of view, there is no insight into their composition in terms of the structural properties of the molecules recognized by IgM.

Prostate-specific antigen (PSA), a well-known tumor marker for prostate cancer (PCa), has been found complexed with IgM in both benign prostatic hyperplasia (BPH) and cancer [3]. In general, PSA comprises heterogeneous molecules differing in primary structure and in glycan composition. Structural variability exists among PSA forms in serum, seminal plasma, and hyperplastic or cancerous tissues [4-6]. Thus, using different experimental approaches, more than 30 immunoreactive glycoisoforms ranging in molecular mass from 6 to $35 \mathrm{kD}$ have been separated from serum, seminal plasma, or prostate tissue [7-12]. Investigation of the structural heterogeneity of PSA revealed that some distinct forms are more frequently associated with $\mathrm{PCa}$ than with BPH [13-15]. For example, some isoforms of precursor or proPSA are cancer specific, whereas other internally cleaved or nicked (multichain) PSA isoforms are characteristic for BPH $[16,17]$.

This study was aimed at complementing existing data on PSA-IgM by defining molecular species of PSA in circulating immune complexes. Having in mind the oligoreactivity and multivalency of IgM and its preference for carbohydrate antigens, there is the possibility that it can selectively recognize 
known PSA glycoisoforms $[18,19]$. Beyond the clinical relevance for cancer detection, this might be of basic importance, since tumor-associated antigens are known to be targets of immune surveillance $[20,21]$.

Circulating PSA-IgM complexes separated by gel filtration from subjects with BPH and PCa were subjected to onchip immunoaffinity profiling using monoclonal anti-PSA antibodies. The detected molecular species were subsequently analyzed for protein and glycan composition and compared to established PSA forms.

\section{Materials and Methods}

2.1. Chemicals and Reagents. Monoclonal anti-free PSA antibody, clone 8A6 (recognizing epitope I), was purchased from Hy Test (Turku, Finland). Monoclonal anti-PSA antibody, clone 8311 (recognizing free and complexed PSA forms), was from Medix Biochemica (Kauniainen, Finland). Sephacryl S-300 was obtained from Pharmacia Biotech (Uppsala, Sweden). Alkaline phosphatase-conjugated anti-human IgM and p-nitrophenyl phosphate (PNPP) were from Institut Virion $\backslash$ Serion $\mathrm{GmbH}$ (Würzburg, Germany). Broad range SDS-PAGE molecular mass standards and Silver Stain Kit were purchased from Bio-Rad (Hercules, CA, USA). ProteinChip: PS20 (preactivated surface), Q10 (strong anion exchanger), CM10 (weak cation exchanger), sinapinic acid, and ProteinChip all-in-one protein standards II were from Bio-Rad (Hercules, CA, USA). Microwell plates were from NUNC (Roskilde, Denmark) and star-bottom tubes were from Spektar (Čačak, Serbia). IgM concentration was determined using an IgM RID kit (INEP, Serbia). Total PSA (tPSA) and free PSA (fPSA) were quantitated using the appropriate commercially available IRMA PSA assays (INEP, Serbia) according to the manufacturer's instructions.

All other chemicals were reagent grade.

2.2. Serum Samples. Serum samples from subjects with benign prostatic hyperplasia $(\mathrm{BPH})$ and prostate cancer (PCa) seen at INEP, Zemun, for PSA determination as a part of followup, were obtained according to local ethical standards (document GSP/05 and PR030/09, approved by the Institutional Committee of INEP). Diagnoses were confirmed using clinically established protocols based on PSA level, physical examination, ultrasound, and biopsy. Samples of PCa sera were from patients diagnosed with locally advanced and advanced cancers.

Individual serum samples from 18 patients with $\mathrm{PCa}$ and $\mathrm{BPH}$ were randomly used (to reflect intrinsic heterogeneity) to form three pools (each $n=6$ ). PSA concentration ranges of the PCa pools were $15.6-776 \mu \mathrm{g} / \mathrm{L} ; 35.4-987.2 \mu \mathrm{g} / \mathrm{L}$ and $20.7-$ $3063.3 \mu \mathrm{g} / \mathrm{L}$. PSA concentration ranges of the $\mathrm{BPH}$ pools were $0.8-5.1 \mu \mathrm{g} / \mathrm{L} ; 0.4-2.5 \mu \mathrm{g} / \mathrm{L}$ and $0.3-5.1 \mu \mathrm{g} / \mathrm{L}$.

2.3. Separation of PSA-IgM Complexes by Gel Filtration. PSA-IgM complexes were separated as described previously with slight modifications [3]. Serum samples $(400 \mu \mathrm{L})$ were applied to a Sephacryl S-300 column $(1.3 \times 25 \mathrm{~cm})$ equilibrated with $0.05 \mathrm{MPBS}, \mathrm{pH} 7.2$, and eluted with the same buffer and $1 \mathrm{~mL}$ fractions were collected. PSA-IgM or PSA was detected in the expected fractions, using solid phase assays with immobilized monoclonal anti-PSA antibody. Antibodycoated tubes $(1.2 \mu \mathrm{g}$ per tube) were prepared by physical adsorption, overnight at $4^{\circ} \mathrm{C}$. After washing with $0.05 \mathrm{M} \mathrm{PBS}$, $\mathrm{pH} 7.2$, tubes were blocked with $0.5 \%$ casein for $3 \mathrm{~h}$ at $37^{\circ} \mathrm{C}$ and rinsed again.

(a) Gel filtration fractions (fractions 1-20) were added $(100 \mu \mathrm{L})$ to coated tubes and incubated for $4 \mathrm{~h}$ at room temperature (RT). After washing thrice with 0.05 MPBS, $\mathrm{pH}$ 7.2, alkaline phosphatase-conjugated anti-human IgM $(100 \mu \mathrm{L})$ was added and incubated for $2 \mathrm{~h}$ at RT.

Unbound conjugate was washed, and $100 \mu \mathrm{L}$ of pNPP substrate solution was added. The reaction was quenched with $100 \mu \mathrm{L} 1 \mathrm{MNaOH}$ after $30 \mathrm{~min}$. The absorption was measured at $405 \mathrm{~nm}$ using a Wallac 1420 multilabel counter (Perkin Elmer, Monza, Italy). Fractions containing PSA-IgM were concentrated and used for further analysis.

In parallel, the total IgM was detected in void volume gel filtration fractions after direct immobilization on polystyrene microwell plates, using alkaline phosphatase-conjugated antihuman IgM, as described above.

(b) PSA was probed by the addition of corresponding gel filtration fractions $(100 \mu \mathrm{L}$ of fractions $1-30)$ to antiPSA coated tubes following simultaneous incubation with iodinated anti-PSA antibody (300000 cpm/tube), overnight at RT. After washing steps, bound radioactivity was detected on a Wallac Wizard 1470 automatic $\gamma$-counter (Perkin Elmer, Waltham, USA). fPSA peak fractions were concentrated and used for further analysis.

\subsection{Immunoelectrophoresis. Samples of $\operatorname{IgM}$ or PSA-IgM} preparations $(10 \mu \mathrm{L})$ were subjected to immunoelectrophoresis on $1 \%$ agar gel for $1.5 \mathrm{~h}$ at $4^{\circ} \mathrm{C}$. Purified sheep anti-human IgM (INEP, Zemun, Serbia) was used for detection.

\subsection{Profiling of PSA-IgM Complexes by on-Chip Immunoaffin-} ity Chromatography. PSA fractions (PSA-IgM or fPSA) separated by gel filtration were profiled using on-chip immobilized monoclonal anti-PSA antibodies, as described [12].

The corresponding antibody $(5 \mu \mathrm{L})$ was applied to each spot of the preactivated surface protein chip array and incubated in a humid chamber overnight at $4^{\circ} \mathrm{C}$. The spots were washed thrice with $5 \mu \mathrm{L}$ of $0.05 \mathrm{MPBS}, \mathrm{pH} 7.2$ for $1 \mathrm{~min}$ at RT and then blocked with $0.05 \mathrm{M}$ Tris- $\mathrm{HCl}, \mathrm{pH} 8.0$ buffer for $1 \mathrm{~h}$ in a humid chamber at RT. After washing three times using the same procedure, $5 \mu \mathrm{L}$ of examined sample was added to each spot and incubated for $2 \mathrm{~h}$ in a humid chamber at RT. The spots were rinsed again thrice with $0.05 \mathrm{M}$ PBS, pH 7.2, and twice with deionized water. All procedures included shaking $(150 \mathrm{rpm})$. After drying, $1 \mu \mathrm{L}$ of $50 \%$ sinapinic acid in acetonitrile $/ \mathrm{dH}_{2} \mathrm{O} /$ trifluoroacetic acid (50\%/49.9\%/0.1\%) was added to each spot, dried, and then reapplied. Nonspecific binding was monitored using IgM (Figure 1, fraction 10) as control sample.

Protein chips were analyzed by surface-enhanced laser desorption/ionization time of flight mass spectrometry (SELDI-TOF/MS) using the ProteinChip Reader, Series 4000, 


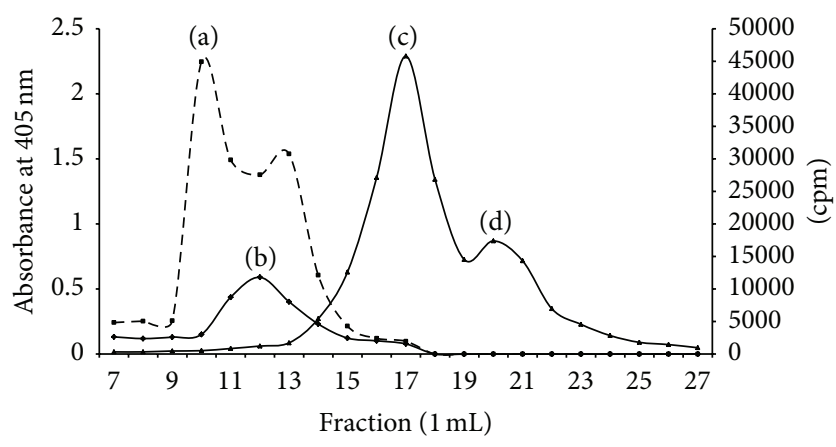

FIGURE 1: Representative PSA-immunogram of pooled human prostate cancer sera. Sephacryl S-300 gel filtration of pooled human PCa sera. The elution was monitored by measuring IgM-reactivity $(--)$ and PSA-reactivity (-) in selected fractions. Fraction volume was $1 \mathrm{~mL}$; cpm: counts per minute. (a) IgM; (b) PSA-IgM; (c) PSAACT (alpha-1-antichymotrypsin); (d) fPSA: free PSA.

Personal Edition (Bio-Rad). All spectra were acquired in $25 \mathrm{kV}$ positive ion acquisition mode, in mass range 2.5$300 \mathrm{kDa}$, and with 8815 laser shots per spot. The laser energy was $6000 \mathrm{~nJ}$. ProteinChip all-in-one protein standards II were used for calibration. All spectra were analyzed using Ciphergen Express Software 3.0 (Bio-Rad, Hercules, CA, USA).

2.6. Profiling of Free PSA Forms by on-Chip Ion-Exchange Chromatography. The spots with incorporated quaternized ammonium groups (Q10, strong anion exchanger) or carboxymethyl groups (CM 10, weak cation exchanger) were prewetted twice with $5 \mu \mathrm{L}$ of $0.05 \mathrm{M}$ Tris- $\mathrm{HCl}, \mathrm{pH} 8$, for $5 \mathrm{~min}$ at RT. After removing the prewetting buffer, $5 \mu \mathrm{L}$ of the examined sample (BPH fPSA or PCa fPSA) was added to each spot and incubated for $1.5 \mathrm{~h}$ in a humid chamber at RT. The spots were rinsed thrice with $0.05 \mathrm{M}$ Tris- $\mathrm{HCl}$, $\mathrm{pH} 8$, and twice with deionized water. All steps included shaking (300 rpm). After drying, $1 \mu \mathrm{L}$ of $50 \%$ sinapinic acid in acetonitrile/ $\mathrm{dH}_{2} \mathrm{O} /$ trifluoroacetic acid $(50 \% / 49.9 \% / 0.1 \%)$ was added to each spot, dried, and then reapplied. Protein chips were analyzed as described above.

\section{Results}

3.1. Separation of PSA-IgM Complexes by Gel Filtration. A representative PSA-immunogram, revealing typical differential elution positions of the main serum fractions, is shown in Figure 1. PSA-IgM (b) is eluted at the trailing edge of the total IgM peak (a). As deduced from its elution position and immunoelectrophoretic appearance (data not shown), PSA-immunoreactivity of IgM complexes seemed to be partly associated with low molecular mass IgM. Gel filtration profiles of several individual runs of pooled PCa and BPH sera gave no striking difference in PSA-IgM peaks, but PSA-ACT (c) and FPSA (d) peaks were considerably lower in $\mathrm{BPH}$, as expected.
3.2. On-Chip Immunoaffinity Chromatography of PSA-IgM Complexes. PSA-IgM preparations from pooled $\mathrm{PCa}$ and $\mathrm{BPH}$ sera were further subjected to on-chip immunoaffinity chromatography, to determine PSA forms in the immune complexes. fPSA fractions were analyzed in parallel as a reference.

Typical mass spectra of PSA-IgM preparations from BPH and PCa sera, profiled using anti-fPSA antibody, are shown in Figure 2 and Table 1. The general pattern was similar, comprising dominant peaks at $17 \mathrm{kDa}$ and $28 \mathrm{kDa}$. The main difference was the presence of a $26889.27 \mathrm{Da}$ species in PCa PSA-IgM absent from BPH PSA-IgM. This appeared as doubly charged ion, which might be related to its structural properties. In comparison to the species at $26.8 \mathrm{kDa}$, the observation of minor peaks at $15.8 \mathrm{kDa}$ or at $16.0 \mathrm{kDa}$ which have close molecular masses was not considered as relevant difference. In addition to anti-fPSA antibody, anti-PSA antibody, recognizing both free and complexed forms, was also used for on-chip chromatography. Comparable spectra were obtained but the intensity and frequency of particular peaks differed slightly. However, this did not additionally contribute to separation and individual characterization of PSA (data not shown).

PSA-immunoreactive forms complexed with $\operatorname{IgM}$, in both $\mathrm{BPH}$ and $\mathrm{PCa}$, overlapped distinct molecular species observed in mass spectra of fPSA fraction (Figure 3). Cluster at $28 \mathrm{kDa}$ was common for both PCa PSA-IgM (Figure 2(a)) and PCa fPSA (Figure $3(\mathrm{a})$ ), whereas the $15.8 \mathrm{kDa}$ species was more abundant in PCa fPSA compared to PCa PSA-IgM.

As for BPH, fPSA fraction displayed a more restricted pattern with the main forms at $27595.81 \mathrm{Da}$ and $28244.95 \mathrm{Da}$ (Figure 3(b)) in contrast to BPH PSA-IgM (Figure 2(b)).

Taking the mass spectra of PSA-IgM preparations obtained from different pools of sera together, subtle differences (some peaks were occasionally present) were noticeable but they all revealed PSA separation into several peaks; that is, they suggested the presence of different PSA glycoisoforms in immune complexes with IgM.

\subsection{Assessment of Glycosylation of PSA Molecular Species} Complexed with IgM. Taking into account the theoretical mass of PSA peptides and their carbohydrate moiety, the observed PSA-IgM species were matched to amino acid sequence and glycan composition of established PSA isoforms (Table 2). The deduced glycan masses of dominant peaks at $17 \mathrm{kDa}$ in BPH PSA-IgM and PCa PSA-IgM ranged between 1121 and $1259 \mathrm{Da}$. They could be attributed to oligomannosidic structures (Man4 or Man5).

The distinct PCa PSA species at 26889.27 is supposed to contain trimannosyl core/paucimannosidic structures (MM), based on the deduced glycans mass of 800.16 Da.

PSA species at $28 \mathrm{kDa}$ are assessed to contain fucosylated or nonfucosylated monosialylated biantennary chain with terminal $\mathrm{N}$-acetylglucosamine $(\mathrm{GnNaF} / \mathrm{NaGnF})$ or galactose $(\mathrm{NaA} / \mathrm{ANa} ; \mathrm{ANaF} / \mathrm{NaAF})$. In addition, the modification through bisecting glucosaminylation ( $\mathrm{NaGnFbi} / \mathrm{GnNaFbi})$ is also suggested. 


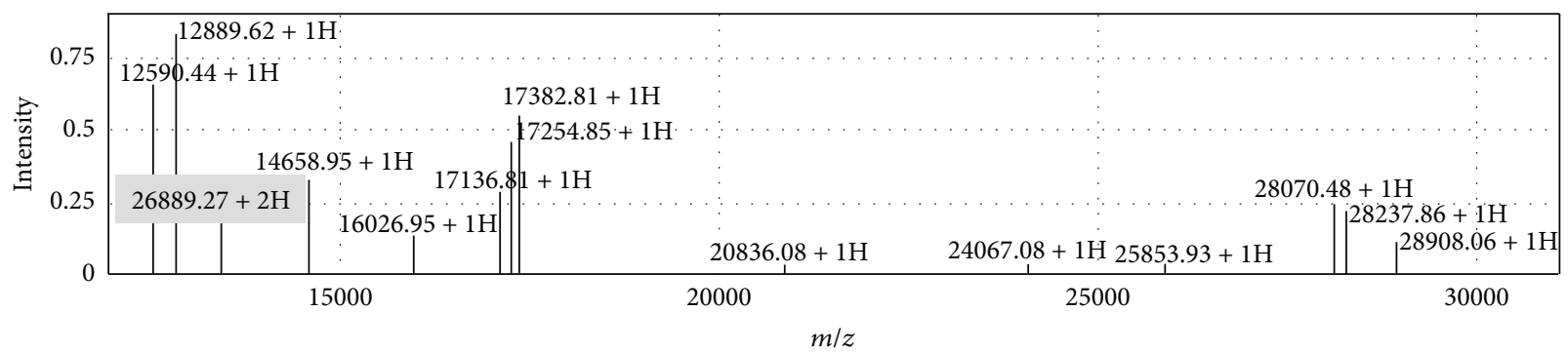

(a)

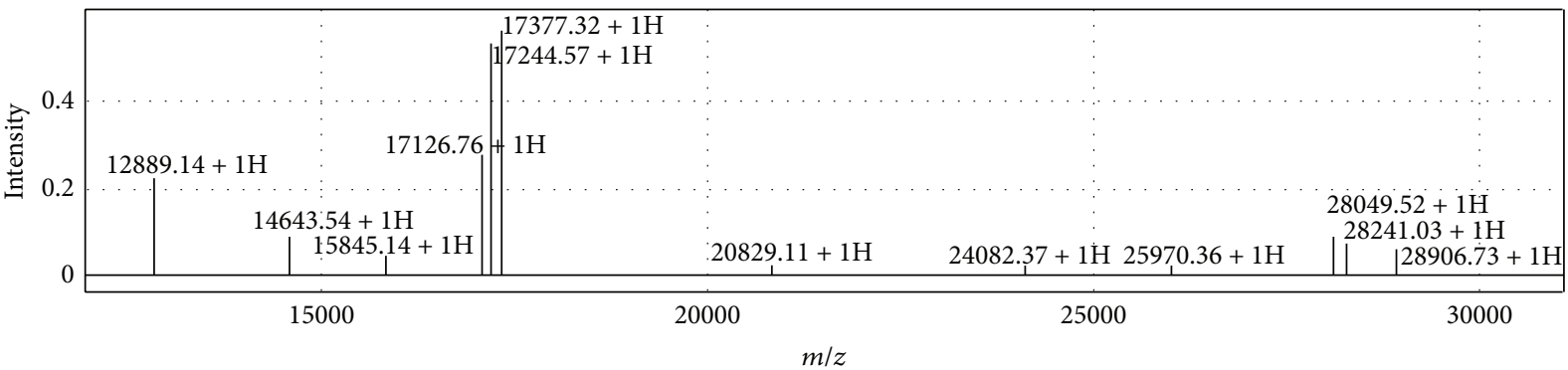

(b)

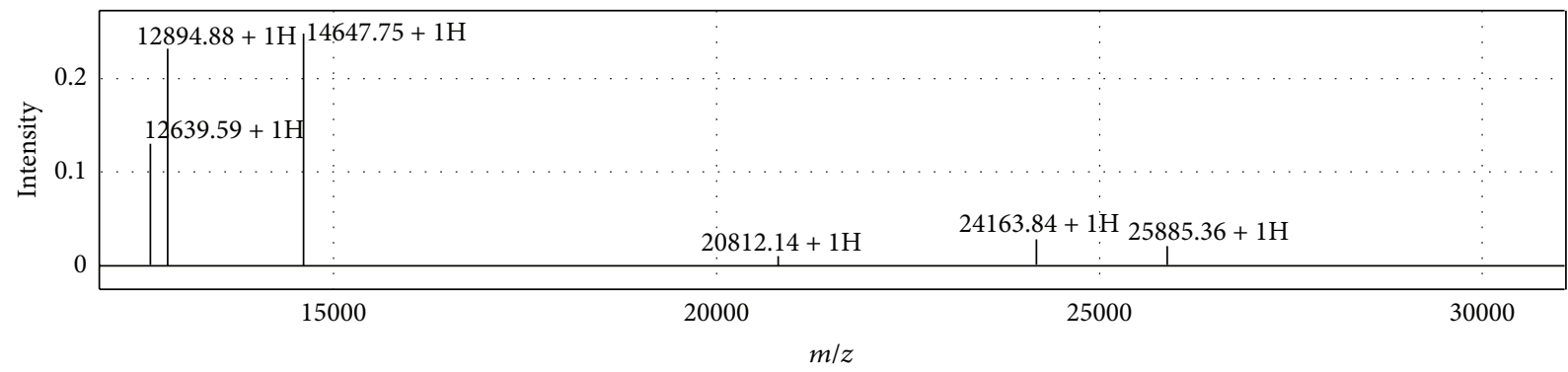

(c)

FIGURE 2: Representative spectra of PSA-immunoreactive species complexed with IgM. On-chip chromatography was performed using antifPSA monoclonal antibody array. Corresponding samples were incubated for $2 \mathrm{~h}$ in a humid chamber at RT. The spots were rinsed, and, after drying, 50\% sinapinic acid was added to each spot, dried, and then reapplied. The spectra were obtained by SELDI-TOF/MS. The $x-$ axis represents the distribution of ions by mass (mass to charge ratio, $m / z$ ) and the $y$-axis gives the signal intensity of the ions. $\mathrm{M}+2 \mathrm{H}$ : double charged ion. (a) PCa PSA-IgM (PSA-IgM from PCa sera); (b) BPH PSA-IgM (PSA-IgM from BPH sera); (c) IgM, control sample. Peaks found on the control chip were considered as nonspecific. The region below $12 \mathrm{kDa}$ is not shown since it was obscured by non-specific binding. Highlighted peak at 26889.27 Da was detected only in PCa samples.

$28.9 \mathrm{kDa}$ peaks in both $\mathrm{BPH}$ and $\mathrm{PCa}$ have estimated glycan mass of $2817 \mathrm{Da}$. This is higher than mass of complete PSA glycan $(2352 \mathrm{Da})$, and it might be assigned to triantennary oligosaccharide chains.

Due to the limited amounts of particular PSA species for direct analysis, the predictions were validated using ionexchange chromatography of uncomplexed PSA as reference. Taking into account, known data on isoelectric point of PSA glycoisoforms ( $\mathrm{pI}$ 6.9-7.7), corresponding samples were separated on anion and cation exchangers at $\mathrm{pH}$ 8.0. The results obtained indicated that incompletely glycosylated PSA $(17 \mathrm{kDa})$ showed weak binding to both ionic exchangers in contrast to sialylated forms $(28 \mathrm{kDa})$ which bound strongly to anionic exchanger (Figure 4). Thus, the observed charge heterogeneity of PSA molecular species was in agreement with their predicted various degrees of glycosylation.

\section{Discussion}

According to the observed molecular masses, PSA has been classified into two more or less heterogeneous groups of glycosylated (gp28, gp22, gp18, and gp12) or nonglycosylated (p26-full length nonglycosylated PSA, p20, p16, p10, and p6) peptides [8]. Lower molecular mass PSA results from protein cleavage at several different sites, among which major forms comprise those cuts at either Lys182/Ser183 or Lys145/Ser146 or at both residues $[4,16]$.

Our results indicate that the major molecular species of PCa PSA and BPH PSA found complexed with IgM corresponded to gp18, which is glycosylated PSA amino acid residues $1-145$. In addition, minor forms corresponding to the entire mature glycosylated PSA species, that is, gp28, were also observed. 
TABLE 1: Summary of PSA-immunoreactive species complexed with IgM in PCa and BPH serum pools.

\begin{tabular}{|c|c|c|c|c|c|}
\hline \multirow{2}{*}{ Sample } & \multicolumn{3}{|c|}{ PSA $m / z$} & \multirow{2}{*}{$\begin{array}{c}\text { Mean (SD) } \\
m / z\end{array}$} & \multirow{2}{*}{$\begin{array}{c}\text { Peak } \\
\text { frequency }(\%)\end{array}$} \\
\hline & P I & P II & P III & & \\
\hline \multirow{8}{*}{$\mathrm{PCa}$} & 16029.95 & 16021.78 & 16150.58 & $16067.44(72.12)$ & 100 \\
\hline & 17136.81 & 17142.65 & & $17139.73(4.13)$ & 66 \\
\hline & 17254.85 & 17214.10 & 17261.33 & $17243.43(25.60)$ & 100 \\
\hline & 17382.81 & 17381.47 & 17378.29 & $17380.86(2.32)$ & 100 \\
\hline & 26889.27 & 26894.18 & & $26891.72(3.47)$ & 66 \\
\hline & 28070.48 & 28058.44 & 28078.26 & $28069.06(9.99)$ & 100 \\
\hline & 28237.86 & 28210.13 & 28282.17 & $28243.39(6.34)$ & 100 \\
\hline & 28908.06 & 28875.31 & 28972.21 & $28918.53(49.29)$ & 100 \\
\hline \multirow{7}{*}{$\mathrm{BPH}$} & 15845.14 & 16054.60 & & & $33(33)$ \\
\hline & 17126.76 & 17142.41 & 17136.95 & $17135.37(7.94)$ & 100 \\
\hline & 17244.57 & 17261.52 & 17216.50 & $17240.76(22.74)$ & 100 \\
\hline & 17377.32 & 17396.20 & 17384.40 & $17385.97(9.54)$ & 100 \\
\hline & 28049.52 & 28095.72 & 28074.06 & $28073.10(23.11)$ & 100 \\
\hline & 28241.03 & 28289.75 & 28227.28 & $28252.69(32.83)$ & 100 \\
\hline & 28906.73 & 28926.09 & 28923.16 & $28918.66(10.44)$ & 100 \\
\hline
\end{tabular}

SD: standard deviation, $m / z$ : mass to charge ratio, and P: pool $(n=6)$. Frequency of peaks observed when analyzed individually in each pool (I-III).

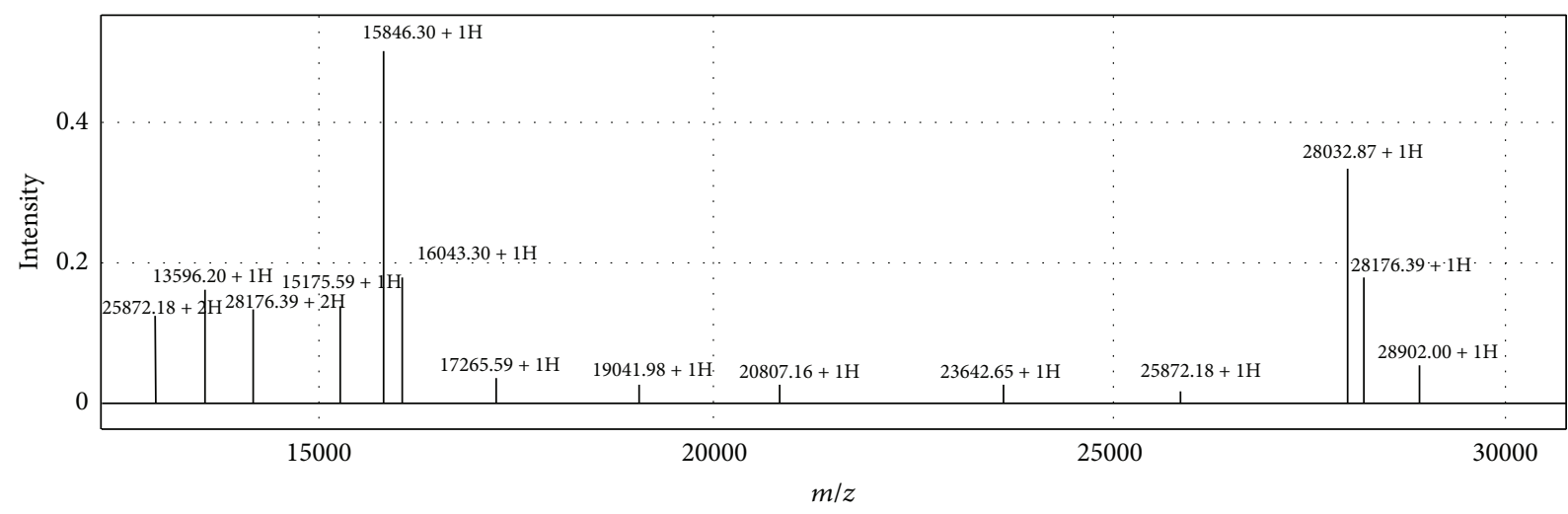

(a)

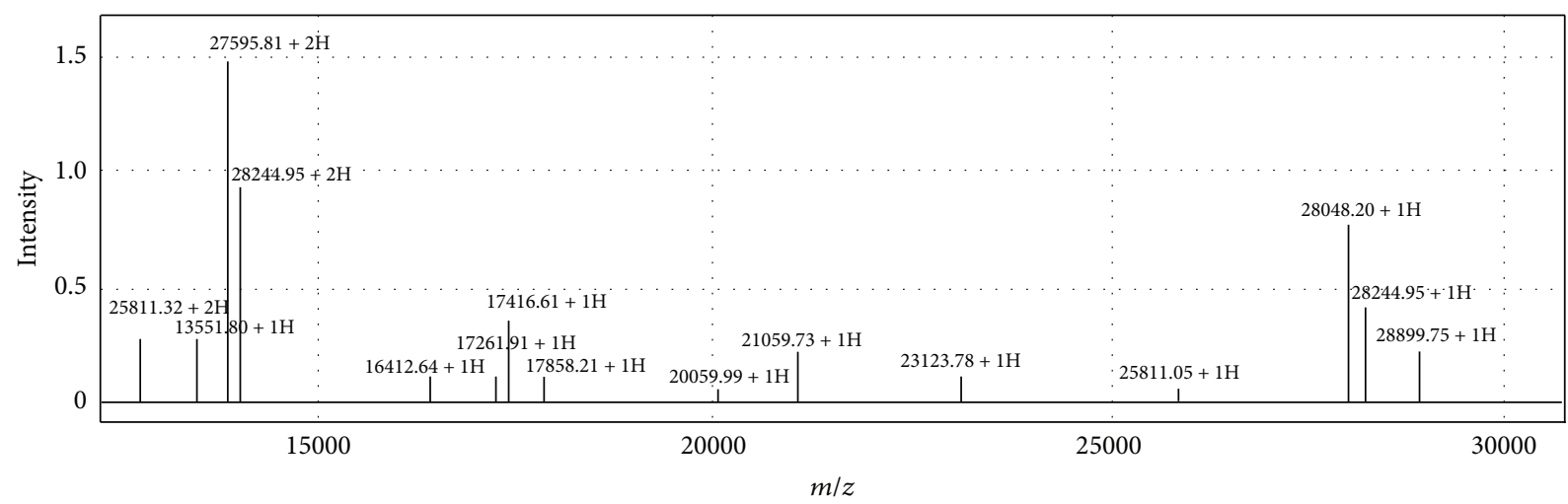

(b)

FIGURE 3: Representative spectra of free PSA-immunoreactive species. On-chip chromatography of free PSA was performed using anti-fPSA monoclonal antibody array. The $x$-axis represents the distribution of ions by mass (mass to charge ratio, $m / z$ ) and the $y$-axis shows signal intensity of the ions. $\mathrm{M}+2 \mathrm{H}$ : double charged form. (a) PCa fPSA (free PSA from PCa sera); (b) BPH fPSA (free PSA from BPH sera). 
TABLE 2: Assessment of glycosylation of PSA molecular species complexed with IgM.

\begin{tabular}{|c|c|c|c|c|c|c|c|c|}
\hline \multirow{3}{*}{$\begin{array}{l}\text { PSA amino acid } \\
\text { sequence theoretical } \\
\text { mass }^{1}\end{array}$} & \multirow{3}{*}{ PSA group ${ }^{2}$} & \multirow{2}{*}{\multicolumn{2}{|c|}{$\begin{array}{c}\text { PSA } \\
\text { Observed mass }\end{array}$}} & \multirow{2}{*}{\multicolumn{2}{|c|}{$\begin{array}{l}\text { PSA glycan }(\mathrm{G}) \\
\text { Calculated mass }{ }^{3}\end{array}$}} & \multirow{3}{*}{$\begin{array}{l}\text { Theoretical } \\
\text { mass }\end{array}$} & \multicolumn{2}{|l|}{ Glycan (G) } \\
\hline & & & & & & & \multirow{2}{*}{ Putative composition ${ }^{4}$} & \multirow{2}{*}{ Structure } \\
\hline & & $\mathrm{PCa}$ & $\mathrm{BPH}$ & $\mathrm{PCa}$ & BPH & & & \\
\hline \multirow{3}{*}{$1-145(16123.50)+\mathrm{G}$} & \multirow{3}{*}{ gp18 } & 17136.81 & 17126.76 & 1013.31 & 1003.26 & \multirow{2}{*}{1054.96} & \multirow{2}{*}{$\operatorname{Man} 4$} & \multirow{3}{*}{$\square-a$} \\
\hline & & 17254.85 & 17244.57 & 1131.35 & 1121.07 & & & \\
\hline & & 17382.81 & 17377.32 & 1259.31 & 1253.82 & 1217.11 & Man5 & \\
\hline \multirow{7}{*}{$1-237(26089.11)+G$} & \multirow{7}{*}{ gp28 } & & & & & 730.69 & & \\
\hline & & 26889.27 & & 800.16 & & 892.82 & MM & \\
\hline & & 28070.48 & 28049.52 & 1981.37 & 1960.41 & 1899.00 & $\mathrm{GnNaF} / \mathrm{NaGnF}$ & \\
\hline & & & & & & 1933.70 & $\mathrm{NaA} / \mathrm{ANa}$ & \\
\hline & & 28237.86 & 28241.03 & 2148.75 & 2151.92 & 2061.00 & $\mathrm{ANaF} / \mathrm{NaAF}$ & \\
\hline & & & & & & 2120.80 & $\mathrm{NaGnFbi/GnNaFbi}$ & $T^{-}$ \\
\hline & & 28908.06 & 28906.73 & 2818.95 & 2817.62 & 2862.60 & $\mathrm{Na}[\mathrm{NaNa}] /[\mathrm{NaNa}] \mathrm{Na}$ & $\square$ \\
\hline
\end{tabular}

\footnotetext{
${ }^{1}$ Theoretical average masses of PSA amino acid sequences were obtained using PeptideMass software (http://web.expasy.org/peptide_mass/peptide-massdoc.html).

${ }^{2}$ According to Isono et al., 2002 [8].

${ }^{3}$ PSA glycan mass was calculated as the difference between observed PSA mass and theoretical mass of corresponding PSA protein sequence.

${ }^{4}$ Putative glycan compositions were proposed based on established complete PSA glycan chain (NaNaF, 2352.16 Da). It is depicted using the nomenclature of the Consortium for Functional Glycomics.

$+\mathrm{G}$ : glycosylation.

The blue square: $\mathrm{Gn}$ (N-acetylglucosamine); the green circle: $\mathrm{M}$ or Man (mannose); the yellow circle: A (galactose); the red triangle: $\mathrm{F}$ (fucose); the purple diamond: $\mathrm{Na}$ (sialic acid); bi: (bisected).
}

PSA is defined as a $28.430 \mathrm{Da}$ glycoprotein containing $8 \%$ of carbohydrates mainly as a biantennary N-linked oligosaccharide of $\mathrm{N}$-acetyllactosamine type with sialic acid group at the end of each of the two branches [22]. However, available data indicate that there is pronounced heterogeneity under both normal and pathological conditions. PSA isoforms differ, generally, in sialic acid content as well as outer chain features [23-25]. Besides biantennary chains, monoantennary sugar chains and peripheral structures composed only of $\mathrm{N}$-acetylhexosamine residues also occur.

The results obtained in this study indicated that PSA species complexed with IgM mostly contained incomplete glycan chain lacking sialic acid. Thus, the main PSA species complexed with IgM are assessed to have oligomannosidic structure in both BPH and PCa. In addition, paucimannosidic structure is assigned to PCa PSA-IgM.

Alterations in PSA glycosylation were reported in both $\mathrm{BPH}$ and PCa [26-30]. Specifically, PSA forms with reduced sialylation, fucosylation changes, and increased pI were found in PCa.

As for unusual mannosidic structures found in PSA-IgM, they can be correlated with glycans of PSA isoform designated as PSA-A ${ }^{\prime}$ [24]. It represented a minor fraction, that is, only $0.6 \%$ of the total PSA isoforms, and comprised four PSA species ranging from 26808.9 to $27295.8 \mathrm{Da}$.
It seems that newly found IgM-bound isoforms could have diagnostic potential to distinguish $\mathrm{PCa}$ and $\mathrm{BPH}$, but with no advantage in comparison to other established parameters (total PSA-IgM or fPSA/PSA). Due to intrinsic heterogeneity of total PSA-IgM and complexity of the method applied, their possible relevance should be confirmed by testing different types of samples, using different experimental designs suitable for clinical use. However, the finding of distinct IgM-bound PSA forms may be of interest in light of recent data indicating paucimannose as marker of human cancers [31,32].

In contrast to these poorly glycosylated PSA species found in complex with IgM, the main fPSA species contained predominantly mono- or disialylated biantennary chains. The fPSA fraction was used as the reference, because it is supposed to comprise an abundant species that can be recognized by IgM. PSA complexed with ACT was not considered relevant for this interaction, since it predominantly contains covalently bound enzymatically active PSA.

PSA glycosylation is very important for its biomarker potential and this was investigated in a number of studies under diverse physiological and pathological conditions [26]. Generally, an examination of PSA glycosylation, especially numerous PSA species/glycoforms, is faced with the problem of small amounts, preventing isolation in sufficient quantity 

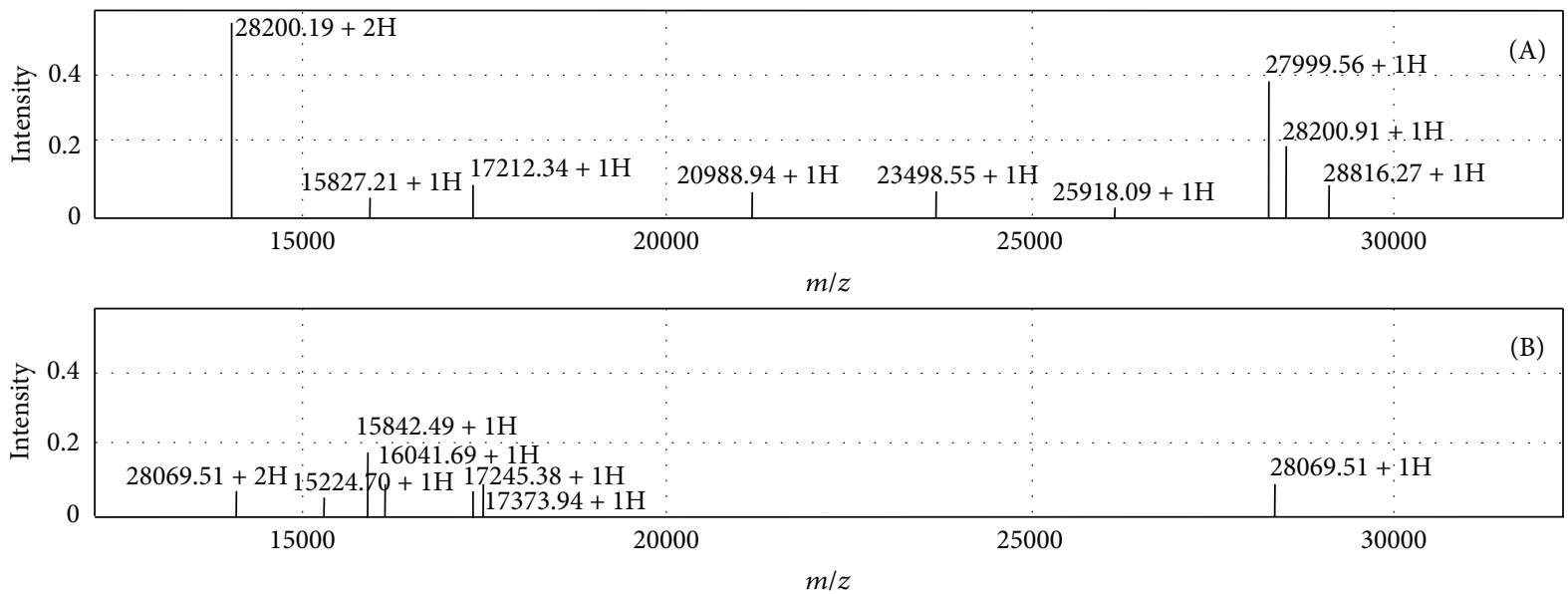

(a)
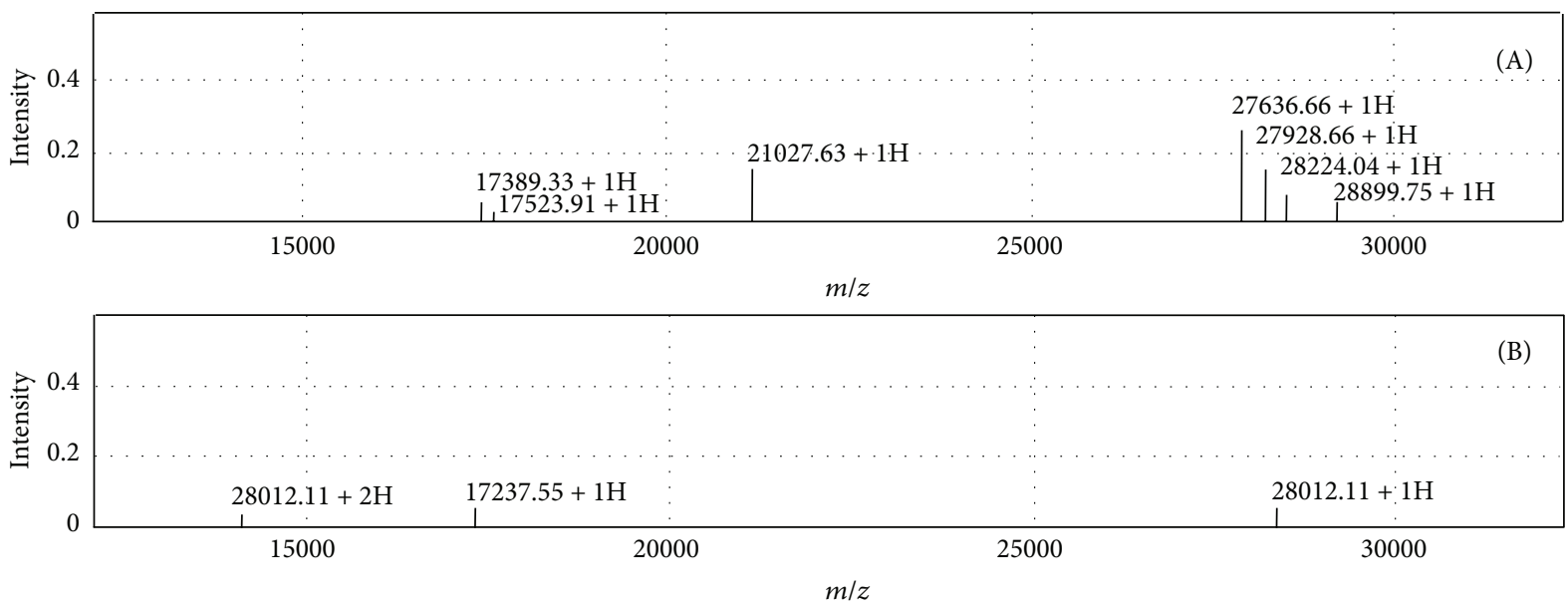

(b)

FIGURE 4: Representative spectra of free PSA isoforms. On-chip ion-exchange chromatography of PCa fPSA (a) and BPH fPSA (b) was performed on anion exchanger, Q10 (A), and cation exchanger, CM10 (B), array at $\mathrm{pH}$. The $x$-axis represents the distribution of ions by mass (mass to charge ratio, $m / z$ ) and the $y$-axis gives the signal intensity of the ions. $\mathrm{M}+2 \mathrm{H}$ : double charged ion. Peaks corresponding to PSA-immunoreactive species are considered as relevant.

for glycan analysis. In addition, the limitation is related to the known influence of both glycan and peptide on PSA behavior during electrophoretic and chromatographic separations.

Using data on the PSA standard preparation obtained by mass spectrometry, assessment of PSA glycosylation by calculation of glycan mass has already been proved relevant [24]. In this study, this was combined with on-chip immunoaffinity chromatography and ion-exchange chromatography, which enables simultaneous sensitive detection of various PSA glycoforms. Thus, despite the differences in methods and detection systems used for this analysis and those reported in the reference literature, generalizations about the composition of PSA isoforms, based on congruence in patterns of protein and glycan moieties, can be made. Differences between calculated and theoretical masses extended to $0.1 \mathrm{kDa}$, which may be due to common ion adducts observed in mass spectrometry. However, for the PSA species at 28.9 Da, the difference was significant. PSA species having masses higher than $28.4 \mathrm{kDa}$ are also reported in seminal plasma and serum, but they are not characterized in detail $[6,8,11,12]$. Their relation to PSA forms containing multiantennary chains or to pro-PSA, as suggested by some authors, cannot be confirmed or denied.

It is known that the structural integrity of PSA significantly influences its biological activity and ability to interact with different molecules. As the first line of defense, IgM has a special position in the immune system and the discovery of PSA-IgM complexes opens an interesting point beyond its biomarker potential. Is the binding of modified carbohydrates, as in the case of PSA, a general principle, not only of basic, but also of clinical interest, for instance, in relation to cancer immunotherapy? Different active and passive vaccines, including carbohydrate-based vaccines to aberrant glycostructures, are under clinical trials but have had limited success at proof of principle and efficacy stages [33]. Characteristic PSA glycoforms bound to IgM, as emerged from this study, direct further investigations to 
structural comparison of different tumor markers found in immune complexes and their free forms and hold promise for advancement of their use as targets for cancer detection and therapy.

\section{Conclusions}

The results obtained indicated distinct differences in immuno-complexed PCa PSA compared to BPH PSA, although both were predominantly composed of nonsialylated incompletely glycosylated molecular species. It poses the question of the possible role of glycosylation as a framework for immunoreactivity and immunoediting, representing a mechanism for early elimination or inactivation of aberrant cancer molecules and cells.

\section{Acknowledgment}

This work is supported by the Ministry of Education, Science and Technological Development of Serbia, project code 173010.

\section{References}

[1] L. Beneduce, F. Castaldi, M. Marino et al., "Improvement of liver cancer detection with simultaneous assessment of circulating levels of free alpha-fetoprotein (AFP) and AFP-IgM complexes," International Journal of Biological Markers, vol. 19, no. 2, pp. 155159, 2004.

[2] L. Beneduce, F. Castaldi, M. Marino et al., "Squamous cell carcinoma antigen-immunoglobulin $\mathrm{M}$ complexes as novel biomarkers for hepatocellular carcinoma," Cancer, vol. 103, no. 12, pp. 2558-2565, 2005.

[3] L. Beneduce, T. Prayer-Galetti, A. M. G. Giustinian et al., "Detection of prostate-specific antigen coupled to immunoglobulin M in prostate cancer patients," Cancer Detection and Prevention, vol. 31, no. 5, pp. 402-407, 2007.

[4] W.-M. Zhang, J. Leinonen, N. Kalkkinen, B. Dowell, and U.-H. Stenman, "Purification and characterization of different molecular forms of prostate-specific antigen in human seminal fluid," Clinical Chemistry, vol. 41, no. 11, pp. 1567-1573, 1995.

[5] A. M. Ward, J. W. F. Catto, and F. C. Hamdy, "Prostate specific antigen: biology, biochemistry and available commercial assays," Annals of Clinical Biochemistry, vol. 38, no. 6, pp. 633651, 2001.

[6] Á. Végvári, M. Rezeli, C. Sihlbom et al., "Molecular microheterogeneity of prostate specific antigen in seminal fluid by mass spectrometry," Clinical Biochemistry, vol. 45, no. 4-5, pp. 331-338, 2012.

[7] K. Jung, B. Brux, M. Lein et al., "Molecular forms of prostatespecific antigen in malignant and benign prostatic tissue: biochemical and diagnostic implications," Clinical Chemistry, vol. 46, no. 1, pp. 47-54, 2000.

[8] T. Isono, T. Tanaka, S. Kageyama, and T. Yoshiki, "Structural diversity of cancer-related and non-cancer-related prostatespecific antigen," Clinical Chemistry, vol. 48, no. 12, pp. 21872194, 2002.

[9] K. Jung, J. Reiche, A. Boehme et al., "Analysis of subforms of free prostate-specific antigen in serum by two-dimensional gel electrophoresis: potential to improve diagnosis of prostate cancer," Clinical Chemistry, vol. 50, no. 12, pp. 2292-2301, 2004.

[10] A. Sarrats, R. Saldova, J. Comet et al., "Glycan characterization of PSA 2-DE subforms from serum and seminal plasma," OMICS, vol. 14, no. 4, pp. 465-474, 2010.

[11] Á. Végvári, M. Rezeli, C. Welinder et al., "Identification of prostate-specific antigen (PSA) isoforms in complex biological samples utilizing complementary platforms," Journal of Proteomics, vol. 73, no. 6, pp. 1137-1147, 2010.

[12] M. M. Kosanovic, S. R. Goc, G. S. Potpara, and M. M. Jankovic, "On chip immuno-affinity profiling of cancer- and benign hyperplasia-associated free prostate specific antigen," Disease Markers, vol. 31, no. 2, pp. 111-118, 2011.

[13] P. R. Huber, H.-P. Schmid, G. Mattarelli, B. Strittmatter, G. J. Van Steenbrugge, and A. Maurer, "Serum free prostate specific antigen: isoenzymes in benign hyperplasia and cancer of the prostate," Prostate, vol. 27, no. 4, pp. 212-219, 1995.

[14] H. Hilz, J. Noldus, P. Hammerer, F. Buck, M. Luck, and H. Huland, "Molecular heterogeneity of free PSA in sera of patients with benign and malignant prostate tumors," European Urology, vol. 36, no. 4, pp. 286-292, 1999.

[15] J. P. Charrier, C. Tournel, S. Michel, S. Comby, C. JolivetReynaud, J. Passagot et al., "Differential diagnosis of prostate cancer and benign prostate hyperplasia using two-dimensional electrophoresis," Electrophoresis, vol. 22, no. 9, pp. 1861-1866, 2001.

[16] S. D. Mikolajczyk, K. M. Marker, L. S. Millar et al., "A truncated precursor form of prostate-specific antigen is a more specific serum marker of prostate cancer," Cancer Research, vol. 61, no. 18, pp. 6958-6963, 2001.

[17] S. D. Mikolajczyk, L. S. Millar, T. J. Wang et al., “BPSA, a specific molecular form of free prostate-specific antigen, is found predominantly in the transition zone of patients with nodular benign prostatic hyperplasia," Urology, vol. 55, no. 1, pp. 41-45, 2000.

[18] H. P. Vollmers and S. Brändlein, "The "early birds": natural IgM antibodies and immune surveillance," Histology and Histopathology, vol. 20, no. 3, pp. 927-937, 2005.

[19] H. P. Vollmers and S. Brändlein, "Natural IgM antibodies: from parias to parvenus," Histology and Histopathology, vol. 21, no. 12, pp. 1355-1366, 2006.

[20] G. P. Dunn, A. T. Bruce, H. Ikeda, L. J. Old, and R. D. Schreiber, "Cancer immunoediting: from immunosurveillance to tumor escape," Nature Immunology, vol. 3, no. 11, pp. 991-998, 2002.

[21] G. P. Dunn, L. J. Old, and R. D. Schreiber, "The three Es of cancer immunoediting," Annual Review of Immunology, vol. 22, pp. 329-360, 2004.

[22] A. Bélanger, H. Van Halbeek, H. C. B. Graves et al., "Molecular mass and carbohydrate structure of prostate specific antigen: studies for establishment of an international PSA standard," Prostate, vol. 27, no. 4, pp. 187-197, 1995.

[23] T. Okada, Y. Sato, N. Kobayashi et al., "Structural characteristics of the N-glycans of two isoforms of prostate-specific antigens purified from human seminal fluid," Biochimica et Biophysica Acta, vol. 1525, no. 1-2, pp. 149-160, 2001.

[24] J. M. Mattsson, L. Valmu, P. Laakkonen, U.-H. Stenman, and H. Koistinen, "Structural characterization and anti-angiogenic properties of prostate-specific antigen isoforms in seminal fluid," Prostate, vol. 68, no. 9, pp. 945-954, 2008.

[25] K. Y. White, L. Rodemich, J. O. Nyalwidhe et al., "Glycomic characterization of prostate-specific antigen and prostatic acid 
phosphatase in prostate cancer and benign disease seminal plasma fluids," Journal of Proteome Research, vol. 8, no. 2, pp. 620-630, 2009.

[26] T. Vermassen, M. M. Speeckaert, N. Lumen, S. Rottey, and J. R. Delanghe, "Glycosylation of prostate specific antigen ant its potential diagnostic applications," Clin Chim Acta, vol. 413, no. 19-20, pp. 1500-1505, 2012.

[27] S. Prakash and P. W. Robbins, "Glycotyping of prostate specific antigen," Glycobiology, vol. 10, no. 2, pp. 173-176, 2000.

[28] R. Peracaula, G. Tabarés, L. Royle et al., "Altered glycosylation pattern allows the distinction between prostate-specific antigen (PSA) from normal and tumor origins," Glycobiology, vol. 13, no. 6, pp. 457-470, 2003.

[29] M. M. Janković and M. M. Kosanović, "Glycosylation of urinary prostate-specific antigen in benign hyperplasia and cancer: assessment by lectin-binding patterns," Clinical Biochemistry, vol. 38, no. 1, pp. 58-65, 2005.

[30] M. M. Kosanović and M. M. Janković, "Sialylation and fucosylation of cancer-associated prostate specific antigen," Journal of Balkan Union of Oncology, vol. 10, no. 2, pp. 247-250, 2005.

[31] C. I. Balog, K. Stavenhagen, W. L. Fung, C. A. Koeleman, L. A. McDonnell, A. Verhoeven et al., "N-glycosylation of colorectal cancer tissues: a liquid chromatography and mass spectrometry-based investigation," Molecular \& Cellular Proteomics, vol. 11, pp. 571-585, 2012.

[32] B. Zipser, D. Bello-DeOcampo, S. Diestel, M. H. Tai, and B. Schmitz, "Mannitou monoclonal antibody uniquely recognizes paucimannose, a marker for human cancer, stemness and inflammation," Journal of Carbohydrate Chemistry, vol. 31, no. 4-6, pp. 504-518, 2012.

[33] H. H. Wandall and M. A. Tarp, "Therapeutic cancer vaccines: Clinical trials and applications," in Carbohydrate-Based Vaccines and Immunotherapies, Z. Guo and G. J. Boons, Eds., pp. 333366, John Wiley \& Sons, Hoboken, NJ, USA, 2009. 


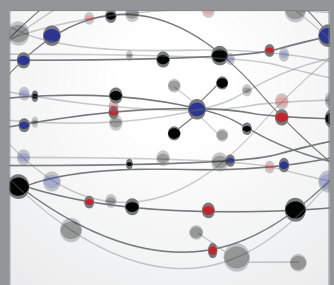

The Scientific World Journal
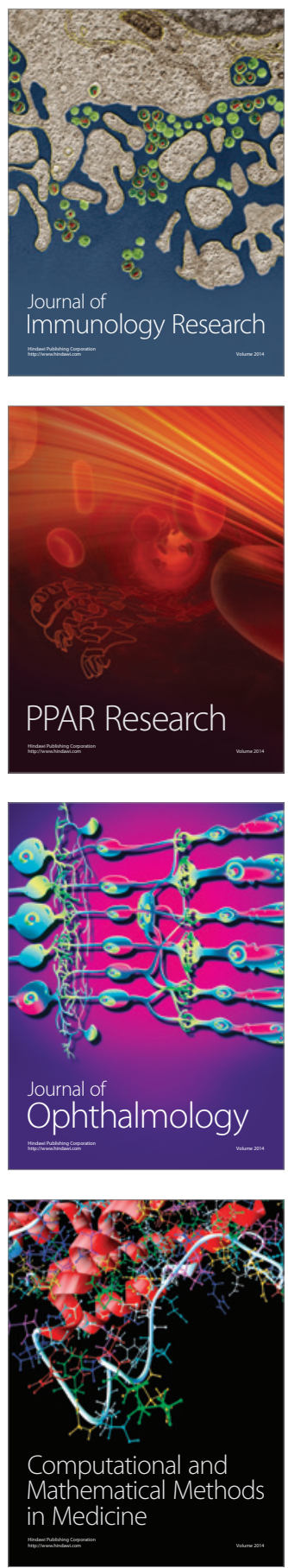

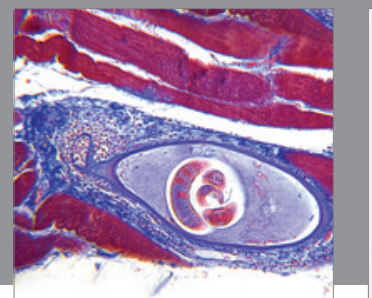

Gastroenterology

Research and Practice
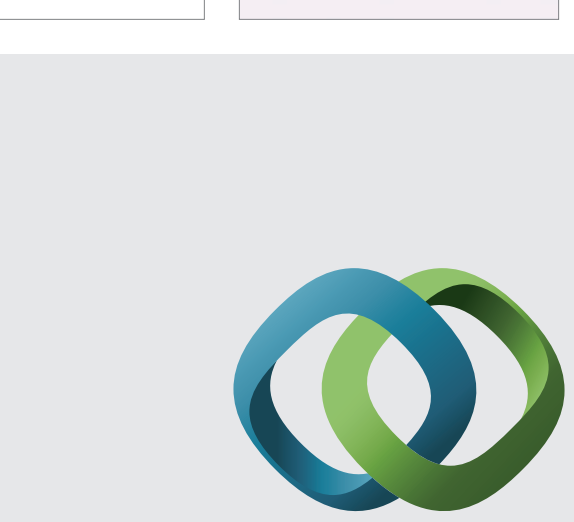

\section{Hindawi}

Submit your manuscripts at

http://www.hindawi.com
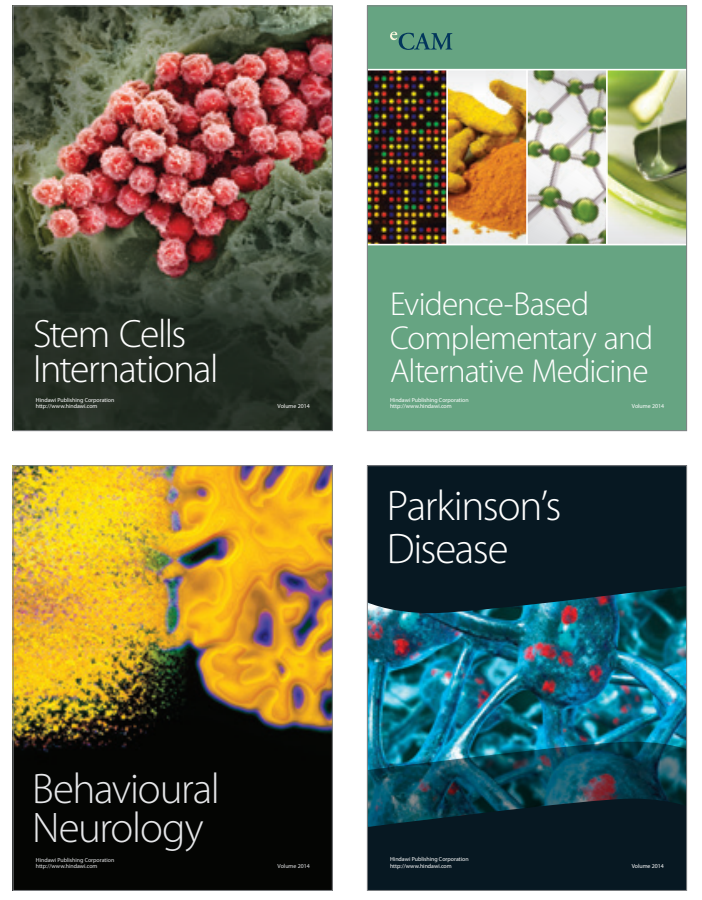
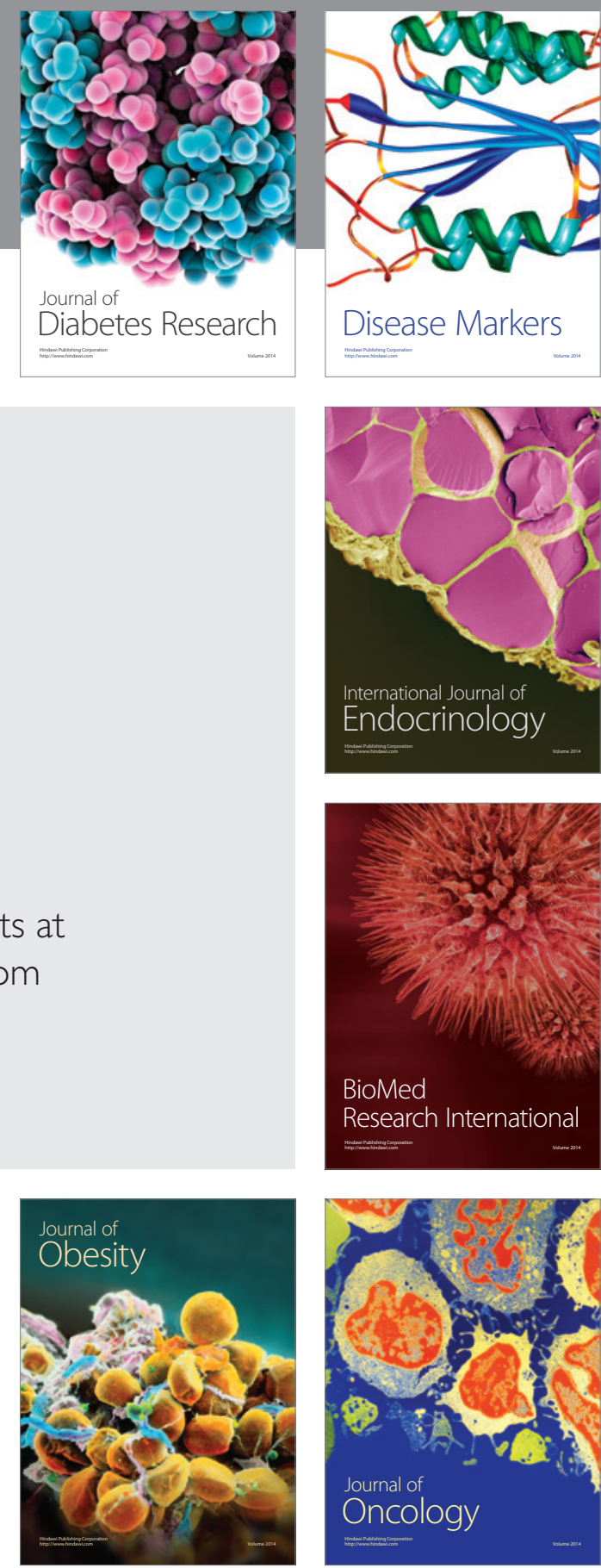

Disease Markers
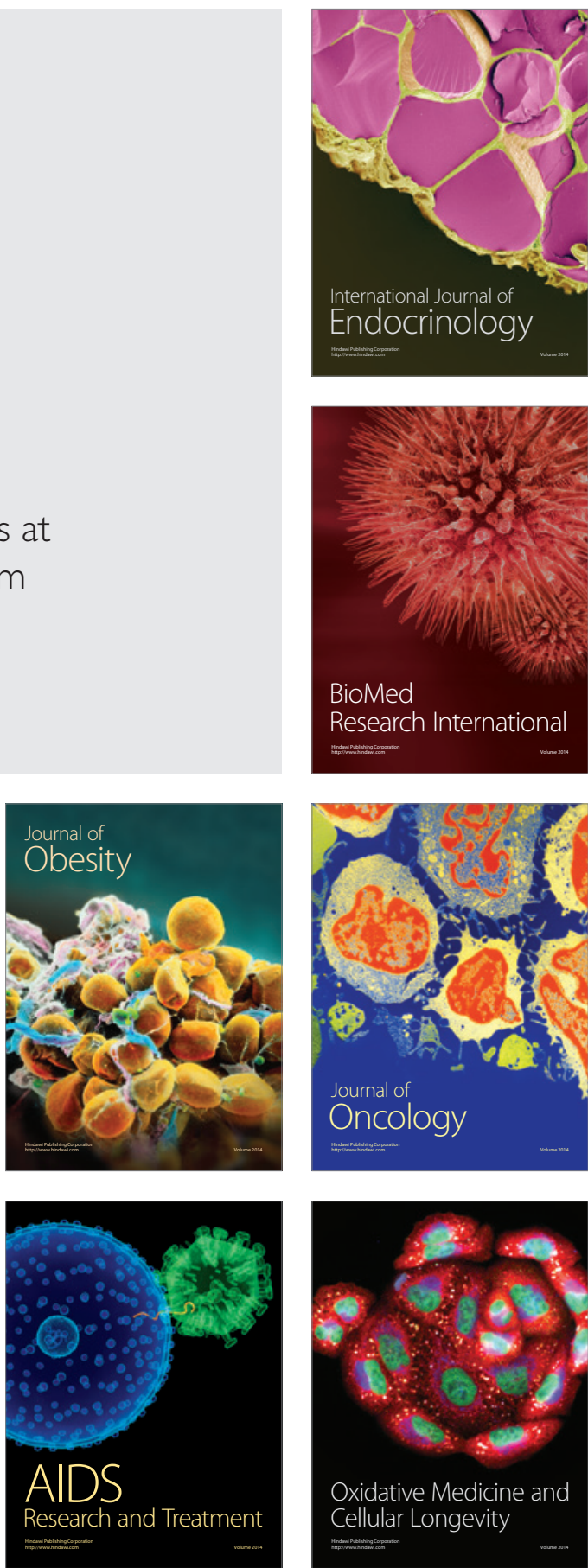